\title{
Nonlinear interaction of charged particles with a free electron gas beyond the random-phase approximation
}

\author{
T. del Río Gaztelurrutia ${ }^{1}$ and J. M. Pitarke ${ }^{2,3}$ \\ ${ }^{1}$ Fisika Aplikatua Saila, Industri eta Telekomunikazio Ingeniarien Goi Eskola Teknikoa, Urkijo Zumarkalea z/g, S-48013 Bilbo, Spain \\ ${ }^{2}$ Materia Kondentsatuaren Fisika Saila, Zientzi Fakultatea, Euskal Herriko Unibertsitatea, 644 Posta kutxatila, \\ 48080 Bilbo, Basque Country, Spain \\ ${ }^{3}$ Donostia International Physics Center (DIPC) and Centro Mixto CSIC-UPV/EHU, Donostia, Basque Country, Spain
}

(Received 7 February 2000; revised manuscript received 14 June 2000)

\begin{abstract}
A nonlinear description of the interaction of charged particles penetrating a solid has become of basic importance in the interpretation of a variety of physical phenomena. Here we develop a many-body theoretical approach to the quadratic decay rate, energy loss, and wake potential of charged particles moving in an interacting free electron gas. Explicit expressions for these quantities are obtained either within the randomphase approximation (RPA) or with full inclusion of short-range exchange and correlation effects. The $Z_{1}^{3}$ correction to the energy loss of ions is evaluated beyond the RPA, in the limit of low velocities.
\end{abstract}

When charged particles pass through a solid, energy can be lost to the medium through various types of elastic and inelastic collision processes. ${ }^{1}$ While at relativistic velocities radiative losses may become important, for moving charged particles in the nonrelativistic regime the energy loss is primarily due to electron-electron $(e-e)$ interactions giving rise to the generation of electron-hole pairs, collective excitations such as plasmons, and inner-shell excitations and ionizations. Energy losses due to nuclear recoil are negligible, unless the projectile velocity is very small compared to the mean speed of electrons in the solid. ${ }^{2}$

The inelastic decay rate of charged particles in a degenerate interacting free electron gas (FEG) has been calculated for many years in the first Born approximation or, equivalently, within linear-response theory. This is a good approximation when the velocity of the projectile is much greater than the average velocity of target electrons. However, in the case of projectiles moving with smaller velocities, nonlinearities have been shown to play a key role in the interpretation of a variety of experiments. Energy-loss measurements have revealed differences, not present within linear-response theory, between the energy loss of protons and antiprotons. ${ }^{3,4}$ Moreover, experimentally observed coherent doubleplasmon excitations ${ }^{5,6}$ cannot be described within linearresponse theory, and nonlinearities may also play an important role in the electronic wake generated by moving ions in a FEG. ${ }^{7}$

Pioneering nonlinear calculations of the electronic energy loss of low-energy ions in an electron gas were performed by Echenique et al. ${ }^{8}$ These authors computed the scattering cross section for a statically screened potential, which was determined self-consistently using density-functional theory (DFT). ${ }^{9}$ These static-screening calculations have recently been extended to velocities approaching the Fermi velocity. ${ }^{10}$ Second-order perturbative calculations, which do not have the limitation of being restricted to low projectile velocities, have been reported by different authors with use of the random-phase approximation (RPA) and by treating the moving charged particle as a prescribed source of energy and momentum. ${ }^{11-15}$
In this paper, we report a many-body theoretical approach to the quadratic decay rate, energy loss, and wake potential of charged particles moving in an interacting FEG. The decay rate is derived from the knowledge of the projectile selfenergy. The energy loss and wake potential are obtained within quadratic response theory. While the first-order contribution to the energy loss may also be obtained from the imaginary part of the projectile self-energy by simply inserting the energy transfer inside the integrand of this quantity, our results indicate that this procedure cannot be generalized to the description of the second-order energy loss. Unless otherwise is stated, we use atomic units throughout, i.e., $e^{2}$ $=\hbar=m_{e}=1$.

We consider the interaction of a moving probe particle of charge $Z_{1}$ and mass $M$ with a FEG of density $n$. The probe particle is assumed to be distinguishable from the electrons in the Fermi gas, which is described by an isotropic homogeneous assembly of interacting electrons immersed in a uniform background of positive charge and volume $V$. Manybody theory shows that the probability for the probe particle to occupy a given excited state of four-momentum $p$ decays exponentially in time with the decay constant ${ }^{16}$

$$
\tau^{-1}=-2 \operatorname{Im} \Sigma_{p}
$$

where $\Sigma_{p}$ represents the particle self-energy.

It is well known that the self-energy cannot be computed by simply evaluating the lowest-order Feynman diagrams, because of severe infrared divergences due to the long-range Coulomb interaction. Instead, one needs to resum electronloop corrections and expand in terms of the dynamically screened interaction. Up to third order in $Z_{1}$, the self-energy of the probe particle can be represented diagrammatically as shown in Fig. 1. The sum of the first two diagrams represents the so-called $G W$ approximation, and the third diagram accounts for $Z_{1}^{3}$ corrections. One finds 

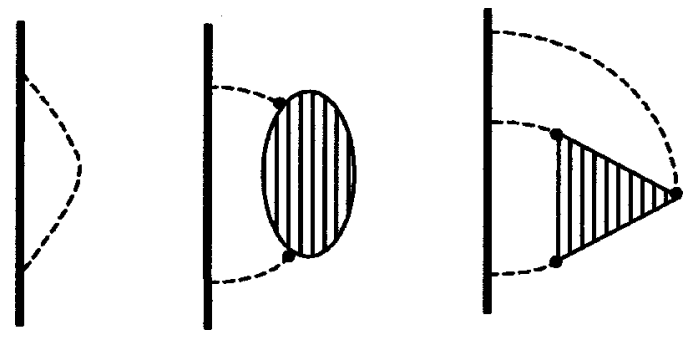

FIG. 1. The probe-particle self-energy, up to third order in $Z_{1}$. Thick solid lines represent the exact probe-particle propagator $i D_{p}$. Dashed lines represent the bare Coulomb interaction $-i v_{\mathbf{q}}$. Twoand three-point loops represent time-ordered density correlation functions $i \chi_{q}$ and $-2 Y_{q_{1}, q_{2}}$, respectively.

$$
\begin{aligned}
\Sigma_{p}= & i Z_{1}^{2} \int \frac{d q^{4}}{(2 \pi)^{4}} v_{\mathbf{q}} D_{p-q}\left[\left(1+\chi_{q} v_{\mathbf{q}}\right)\right. \\
& \left.-2 i Z_{1} \int \frac{d^{4} q_{1}}{(2 \pi)^{4}} D_{p-q_{1}} D_{p-q+q_{1}} Y_{q,-q_{1}} v_{\mathbf{q}_{1}} v_{\mathbf{q}-\mathbf{q}_{1}}\right]
\end{aligned}
$$

where $v_{\mathbf{q}}$ is the Fourier transform of the bare Coulomb potential, $D_{p}$ is the probe-particle propagator,

$$
D_{p}=\frac{1}{p^{0}-\omega_{\mathbf{p}}-\Sigma_{p}+i \eta}
$$

$\omega_{\mathbf{p}}=\mathbf{p}^{2} /(2 M)$ is the noninteracting energy, and $\eta$ is a positive infinitesimal. $\chi_{q}$ and $Y_{q_{1}, q_{2}}$ represent exact time-ordered density correlation functions of the interacting FEG,

$$
\chi_{q}=\frac{1}{V} \sum_{n}\left|\left(\rho_{\mathbf{q}}\right)_{n 0}\right|^{2}\left[\frac{1}{q^{0}+\omega_{0 n}+\mathrm{i} \eta}-\frac{1}{q^{0}-\omega_{0 n}-i \eta}\right]
$$

and

$$
\begin{aligned}
Y_{q_{1}, q_{2}}= & -\frac{1}{2 V} \sum_{n, l}\left[\frac{\left(\rho_{\mathbf{q}_{1}}\right)_{0 n}\left(\rho_{\mathbf{q}_{3}}\right)_{n l}\left(\rho_{\mathbf{q}_{2}}\right)_{l 0}}{\left(q_{1}^{0}+\omega_{0 n}+\mathrm{i} \eta\right)\left(q_{2}^{0}+\omega_{l 0}-i \eta\right)}\right. \\
& +\frac{\left(\rho_{\mathbf{q}_{2}}\right)_{0 n}\left(\rho_{\mathbf{q}_{1}}\right)_{n l}\left(\rho_{\mathbf{q}_{3}}\right)_{l 0}}{\left(q_{2}^{0}+\omega_{0 n}+i \eta\right)\left(q_{3}^{0}+\omega_{l 0}-\mathrm{i} \eta\right)} \\
& \left.+\frac{\left(\rho_{\mathbf{q}_{3}}\right)_{0 n}\left(\rho_{\mathbf{q}_{2}}\right)_{n l}\left(\rho_{\mathbf{q}_{1}}\right)_{l 0}}{\left(q_{3}^{0}+\omega_{0 n}+\mathrm{i} \eta\right)\left(q_{1}^{0}+\omega_{l 0}-i \eta\right)}+\left(q_{2} \rightarrow q_{3}\right)\right],
\end{aligned}
$$

$\left(\rho_{\mathbf{q}}\right)_{n l}$ being the matrix element of the Fourier transform of the electron-density operator, taken between exact manyelectron states of energy $E_{n}$ and $E_{l}, \omega_{n l}=E_{n}-E_{l}$, and $q_{3}$ $=-\left(q_{1}+q_{2}\right)$.

If the probe particle is an ion $(M \gg 1)$, the propagator $D_{p}$ and the energy $p^{0}$ entering Eq. (2) can be safely approximated by the noninteracting propagator $D_{p}^{0}$ and energy $\omega_{\mathbf{p}}$. Recoil can also be neglected, and the introduction of Eq. (2) into Eq. (1) then yields, after some work of rearrangement, the following expression:

$$
\begin{aligned}
\tau^{-1}= & 4 \pi Z_{1}^{2} \int \frac{d q^{4}}{(2 \pi)^{4}} v_{\mathbf{q}} \delta\left(q^{0}-\mathbf{q} \cdot \mathbf{v}\right) \Theta\left(q^{0}\right) \\
& \times\left[-\operatorname{Im} K_{q}+\frac{4}{3} \pi Z_{1} \int \frac{d^{4} q_{1}}{(2 \pi)^{4}} \operatorname{Im} Y_{q,-q_{1}} v_{\mathbf{q}_{1}} v_{\mathbf{q}-\mathbf{q}_{1}}\right. \\
& \left.\times \delta\left(q_{1}^{0}-\mathbf{q}_{1} \cdot \mathbf{v}\right)\right]
\end{aligned}
$$

where $\mathbf{v}$ is the particle velocity, $\Theta(x)$ represents the Heaviside step function, and $K_{q}$ is the so-called test-charge-testcharge inverse dielectric function:

$$
K_{q}=1+\chi_{q} v_{\mathbf{q}} .
$$

The decay rate of Eq. (6) has not been reported before.

In the RPA, density correlation functions are obtained by summing over all ringlike diagrams,

$$
\chi_{q}^{\mathrm{RPA}}=\chi_{q}^{0}+\chi_{q}^{0} v_{\mathbf{q}} \chi_{q}^{R P A}
$$

and

$$
Y_{q_{1}, q_{2}}^{R P A}=K_{q_{1}}^{R P A} Y_{q_{1}, q_{2}}^{0} K_{-q_{2}}^{R P A} K_{-q_{3}}^{R P A},
$$

where $\chi_{q}^{0}$ and $Y_{q_{1}, q_{2}}^{0}$ represent noninteracting density correlation functions. Improvements on the RPA are typically carried out by introducing an effective $e-e$ interaction ${ }^{17}$

$$
\tilde{v}_{q}=v_{\mathbf{q}}\left(1-G_{q}\right),
$$

where $G_{q}$ is the so-called local-field factor accounting for short-range exchange and correlation (xc) effects not present in the RPA. Accordingly, the density correlation functions $\chi_{q}$ and $Y_{q_{1}, q_{2}}$ are found to be of the RPA form, but with all $e-e$ bare Coulomb interactions $v_{\mathbf{q}}$ replaced by $\tilde{v}_{q}$, i.e.,

$$
\chi_{q}=\chi_{q}^{0}+\chi_{q}^{0} \tilde{v}_{q} \chi_{q}
$$

and

$$
Y_{q_{1}, q_{2}}=\widetilde{K}_{q_{1}} Y_{q_{1}, q_{2}}^{0} \widetilde{K}_{-q_{2}} \widetilde{K}_{-q_{3}}
$$

where $\widetilde{K}_{q}$ is the test-charge-electron inverse dielectric function, ${ }^{18,19}$

$$
\widetilde{K}_{q}=1+\chi_{q} \tilde{v}_{q}
$$

In the RPA, $K_{q}$ and $\widetilde{K}_{q}$ coincide.

The potential induced in a uniform FEG by the presence of a recoiless probe particle may be obtained with the use of time-dependent perturbation theory. Keeping terms of first and second order in the external perturbation, one finds

$$
\begin{aligned}
V^{i n d}(\mathbf{r}, t)= & 2 \pi Z_{1} \int \frac{d^{4} q}{(2 \pi)^{4}} \mathrm{e}^{i\left(\mathbf{q} \cdot \mathbf{r}-q^{0} t\right)} v_{\mathbf{q}} \delta\left(q^{0}-\mathbf{q} \cdot \mathbf{v}\right)\left[\left(K_{q}^{R}-1\right)\right. \\
& \left.-2 \pi Z_{1} \int \frac{d^{4} q_{1}}{(2 \pi)^{4}} Y_{q,-q_{1}}^{R} v_{\mathbf{q}_{1}} v_{\mathbf{q}-\mathbf{q}_{1}} \delta\left(q_{1}^{0}-\mathbf{q}_{1} \cdot \mathbf{v}\right)\right]
\end{aligned}
$$

where we have introduced the retarded counterparts of the time-ordered functions $K_{q}$ and $Y_{q,-q_{1}}$ entering Eq. (6). 
The average energy lost per unit length traveled by the probe particle is obtained as the retarding force due to the potential of Eq. (14) induced in the vicinity of the projectile itself. One easily finds

$$
\begin{aligned}
-\frac{d E}{d x}= & 4 \pi Z_{1}^{2} \int \frac{d^{4} q}{(2 \pi)^{4}} q^{0} v_{\mathbf{q}} \delta\left(q^{0}-\mathbf{q} \cdot \mathbf{v}\right) \Theta\left(q^{0}\right) \\
& \times\left[-\operatorname{Im} K_{q}^{R}+2 \pi Z_{1} \int \frac{d^{4} q_{1}}{(2 \pi)^{4}} \operatorname{Im} Y_{q,-q_{1}}^{R} v_{\mathbf{q}_{1}} v_{\mathbf{q}-\mathbf{q}_{1}}\right. \\
& \left.\times \delta\left(q_{1}^{0}-\mathbf{q}_{1} \cdot \mathbf{v}\right)\right] .
\end{aligned}
$$

Both Eq. (6) for the decay rate and Eq. (15) for the energy loss can be derived, within many-body perturbation theory, from the knowledge of the probability for the probe particle to transfer four-momentum $q$ to the FEG. ${ }^{20}$ Nevertheless, this probability cannot be identified with the integrand of Eq. (6), and therefore the energy loss of Eq. (15) cannot be obtained by simply inserting the energy transfer inside the integrand of Eq. (6).

In the framework of time-dependent density-functional theory (TDDFT) ${ }^{21}$ quadratic response theory yields

$$
\begin{gathered}
\chi_{q}^{R}=\chi_{q}^{R, 0}+\chi_{q}^{R, 0}\left(v_{\mathbf{q}}+f_{q}^{x c}\right) \chi_{q}^{R}, \\
Y_{q_{1}, q_{2}}^{R}=\widetilde{K}_{q_{1}}^{R} Y_{q_{1}, q_{2}}^{0} \widetilde{K}_{-q_{2}}^{R} \widetilde{K}_{-q_{3}}^{R}, \\
K_{q}^{R}=1+\chi_{q}^{R} v_{\mathbf{q}},
\end{gathered}
$$

and

$$
\widetilde{K}_{q}^{R}=1+\chi_{q}^{R}\left(v_{\mathbf{q}}+f_{q}^{x c}\right),
$$

where $\chi_{q}^{R, 0}$ and $Y_{q_{1}, q_{2}}^{R, 0}$ represent retarded noninteracting density correlation functions, and $f_{q}^{x c}$ denotes the Fourier transform of

$$
f^{x c}\left(x, x^{\prime}\right)=\frac{\delta V^{x c}([n], x)}{\delta n\left(x^{\prime}\right)},
$$

$V^{x c}([n], x)$ being the exact time-dependent xc potential of TDDFT. ${ }^{21}$ Within the RPA $f_{q}^{x c}=0$, and the introduction of Eqs. (17) and (18) into Eqs. (14) and (15) then yields the results derived in Refs. 11-15.

At this point, we present an application of our formalism, namely, the low-velocity limit of the energy loss with inclusion of short-range xc effects. For low projectile velocities $(v \rightarrow 0)$, only the static $(\omega \rightarrow 0) \quad \chi_{q}^{R, 0}, Y_{q_{1}, q_{2}}^{R, 0}$, and $f_{q}^{x c}$ enter in the evaluation of the energy loss of Eq. (15), which is then easily found to be proportional to the projectile velocity. In particular, in the so-called local-density approximation (LDA), which is rigorous in the long-wavelength limit ( $q$ $\rightarrow 0$ ), one finds

$$
f_{q}^{x c}=\frac{4 \pi}{q_{F}^{2}}\left[\frac{1}{4}-\frac{4 \pi}{q_{F}^{2}} \frac{d^{2} E_{c}}{d n^{2}}\right],
$$

$E_{c}(n)$ being the correlation energy of a uniform electron gas of density $n$.

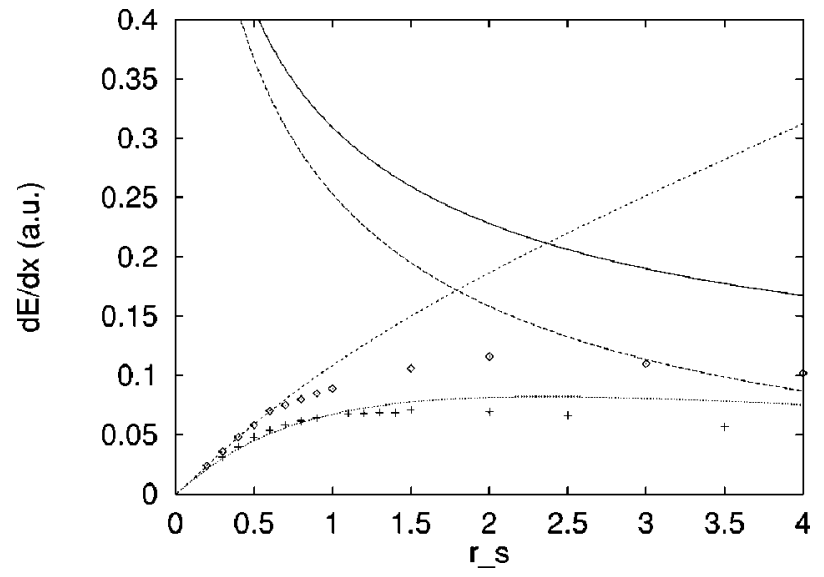

FIG. 2. $Z_{1}^{2}$ and $Z_{1}^{3}$ contributions to the low-velocity electronic stopping power of Eq. (15) divided by the velocity of the projectile, as a function of $r_{s}$, for $Z_{1}=1$. Our full calculations, as obtained with inclusion of LDA exchange and correlation, are represented by solid $\left(Z_{1}^{2}\right.$ contribution) and short-dashed $\left(Z_{1}^{3}\right.$ contribution) lines. Long-dashed $\left(Z_{1}^{2}\right)$ and dotted $\left(Z_{1}^{3}\right)$ lines represent the corresponding results obtained within the RPA $\left(f_{q}^{x c}=0\right)$. The difference (multiplied by a factor of -1 ) between the full nonlinear stopping power for antiprotons, as reported in Ref. 26, and the linear contribution is represented by crosses (xc effects excluded) and rhombs (LDA xc included).

Diffusion Monte Carlo calculations of $\chi_{q}^{0}$ have shown that the LDA static xc kernel of Eq. (21) reproduces correctly the static response for all $q \leqslant 2 q_{F}$. ${ }^{22}$ We have calculated static density correlation functions from Eqs. (16) and (17), with use of the LDA static xc kernel of Eq. (21) and the Perdew-Zunger ${ }^{23}$ parametrization of the quantum Monte Carlo correlation energy $E_{c}(n)$ of Ceperley and Alder. ${ }^{24}$

In Fig. 2 we show linear $\left(\propto Z_{1}^{2}\right)$ and quadratic $\left(\propto Z_{1}^{3}\right)$ contributions to the low-velocity energy loss of Eq. (15) divided by the velocity of the projectile, as a function of the electrondensity parameter $r_{s} .{ }^{25}$ Comparison between our results (solid and short-dashed lines) and those obtained within the RPA (long-dashed and dotted lines) indicates that xc effects become increasingly important as the electron density decreases, the impact of these effects being more pronounced for the $Z_{1}^{3}$ than for the $Z_{1}^{2}$ contribution to the energy loss. As a result, the importance of the quadratic contribution increases when xc effects are included, and for $r_{s} \sim 2.5$ it is equal in magnitude to the linear contribution (within the RPA, linear and quadratic contributions are equal for $r_{s}$ $\sim 5$ ).

The crosses and rhombs in Fig. 2 represent the full nonlinear contribution to the energy loss (difference between the total energy loss and the linear contribution), multiplied by a factor of -1 , as obtained from DFT calculations for antiprotons to all orders in $Z_{1},{ }^{26}$ with xc effects excluded (crosses) and with LDA xc effects included (rhombs). Both full nonlinear calculations are found to agree nicely with our quadratic-response calculations in the high-density limit $\left(r_{s}\right.$ $\rightarrow 0$ ). As the electron density decreases, quadratic-response calculations overestimate the energy loss of antiprotons, especially when $\mathrm{xc}$ effects are included, showing that $\mathrm{xc}$ and nonlinear (beyond $Z_{1}^{3}$ ) effects tend to compensate.

Finally, we note that linear and quadratic contributions to 
the energy loss can be extracted from the small- $Z_{1}$ behavior of full nonlinear DFT calculations, which have been reported in the low-velocity limit. ${ }^{8}$ When $(-d E / d x) / Z_{1}^{2}$ is plotted as a function of $Z_{1}$, the result has a linear $Z_{1}$ dependence at small $Z_{1}$. The intercept and the slope of this curve at $Z_{1}$ $=0$ give linear and quadratic contributions to the full nonlinear energy loss, as shown in Ref. 12, by simply ignoring $\mathrm{xc}$ effects. These authors repeated their full nonlinear DFT calculations with LDA xc included, but were unable to compare them with quadratic-response calculations that included xc effects at the same level of approximation. The results of these quadratic-response calculations are now shown in Fig. 2 . It can be seen that the ratio between xc-included (shortdashed line) and xc-excluded (dotted line) quadratic contri butions to the energy loss is in excellent agreement with the ratios reported in Ref. 12 (see Fig. 7 of this reference) as derived from the $Z_{1}$ dependence of full nonlinear DFT calculations.

In conclusion, we have developed a many-body theoretical approach to the quadratic decay rate, energy loss, and wake potential of charged particles moving in an electron gas, with full inclusion of short-range xc effects. We have shown that in the limit of high electron densities and low projectile charges our calculated quadratic energy loss, which can be extended to the case of larger velocities, reproduces DFT calculations for antiprotons, as long as exchange and correlation are treated at the same level of accuracy.

The authors acknowledge partial support by the University of the Basque Country, the Basque Hezkuntza, Unibertsitate eta Ikerketa Saila, and the Spanish Ministerio de Educación y Cultura.
${ }^{1}$ P. M. Echenique, F. Flores, and R. H. Ritchie, Solid State Phys. 43, 229 (1990).

${ }^{2}$ M. A. Kumakhov and F. F. Komarov, Energy Loss and Ion Ranges in Solids (Gordon and Breach, New York, 1981).

${ }^{3}$ L. H. Andersen et al., Phys. Rev. Lett. 62, 1731 (1989).

${ }^{4}$ S. Moller et al., Phys. Rev. A 56, 2930 (1997).

${ }^{5}$ J. C. Spence and A. E. Spargo, Phys. Rev. Lett. 26, 895 (1971).

${ }^{6}$ P. Schattschneider, F. Fodermayr, and D. S. Su, Phys. Rev. Lett. 59, 724 (1987).

${ }^{7}$ D. S. Gemmell and Z. Vager, in Treatise on Heavy-Ion Science, edited by D. Allan Bromley (Plenum, New York, 1985), Vol. 6, p. 243.

${ }^{8}$ P. M. Echenique, R. M. Nieminem, J. C. Ashley, and R. H. Ritchie, Phys. Rev. A 33, 897 (1986).

${ }^{9}$ P. Hohenberg and W. Kohn, Phys. Rev. 136, B864 (1964); W. Kohn and L. J. Sham, Phys. Rev. 140, A1133 (1965).

${ }^{10}$ A. Salin, A. Arnau, P. M. Echenique, and E. Zaremba, Phys. Rev. B 59, 2537 (1999).

${ }^{11}$ C. C. Sung and R. H. Ritchie, Phys. Rev. A 28, 674 (1983).

${ }^{12}$ C. D. Hu and E. Zaremba, Phys. Rev. B 37, 9268 (1988).

${ }^{13}$ H. Esbensen and P. Sigmund, Ann. Phys. (N.Y.) 201, 152 (1990).

${ }^{14}$ J. M. Pitarke, R. H. Ritchie, and P. M. Echenique, Nucl. Instrum.
Methods Phys. Res. B 79, 209 (1993); J. M. Pitarke, R. H. Ritchie, P. M. Echenique, and E. Zaremba, Europhys. Lett. 24, 613 (1993).

${ }^{15}$ J. M. Pitarke, R. H. Ritchie, and P. M. Echenique, Phys. Rev. B 52, 13883 (1995). The symmmetrized empty three-point function defined in this reference is $M_{q, q_{1}}=-Y_{q,-q_{1}}^{0}$.

${ }^{16}$ G. D. Mahan, Many-Particle Physics (Plenum, New York, 1990).

${ }^{17}$ See, e.g., K. S. Singwi and M. P. Tosi, Solid State Phys. 36, 177 (1981).

${ }^{18}$ L. Kleinman, Phys. Rev. 172, 383 (1968).

${ }^{19}$ L. Hedin and B. I. Lundqvist, J. Phys. C 4, 2064 (1971).

${ }^{20} \mathrm{~T}$. del Río and J. M. Pitarke (unpublished).

${ }^{21}$ M. Petersilka, U. J. Gossmann, and E. K. U. Gross, Phys. Rev. Lett. 76, 1212 (1996)

${ }^{22}$ C. Bowen, G. Sugiyama, and B. J. Alder, Phys. Rev. B 50, 14838 (1994); S. Moroni, D. M. Ceperley, and G. Senatore, Phys. Rev. Lett. 75, 689 (1995).

${ }^{23}$ J. Perdew and A. Zunger, Phys. Rev. B 23, 5048 (1981).

${ }^{24}$ D. M. Ceperley and B. J. Alder, Phys. Rev. Lett. 45, 1196 (1980).

${ }^{25} r_{s}$ is defined by the relation $1 / n_{0}=(4 / 3) \pi r_{s}^{3}$.

${ }^{26}$ I. Nagy, A. Arnau, P. M. Echenique, and E. Zaremba, Phys. Rev. B 40, 11983 (1989). 